\title{
A guide to functional disease for a trauma conscious generation
}

\author{
Author: Andrew Whitfield ${ }^{\mathrm{A}}$
}

In part due to a greater understanding of trauma and its manifestation as disease, the field of functional neurology has seen rapid development over the past decade with the inauguration of the Functional Neurological Disorder Society. Recent developments in our understanding of functional neurology are translatable to other functional disorders and have laid the groundwork for future research opportunities that foundation trainees can contribute towards. At the very least, trainees have a responsibility to be aware of the involuntary nature of these conditions and direct patients towards appropriate help. This guide to functional neurology aims to clear the fog on this collection of poorly recognised conditions so that empathy and understanding can shine through.

KEYWORDS: functional, psychogenic, trauma, foundation, learning

DOI: 10.7861/clinmed.2020-1023

\section{Background}

Foundation trainees may have already recognised functional diagnoses such as irritable bowel, chronic fatigue or fibromyalgia presenting across a variety of specialties. However, it is within functional neurological disease (FND) that the greatest advances in understanding have been made in the past decade with findings translatable across functional medicine. Broadly defined by altered voluntary motor or sensory function, and encompassing conditions such as psychogenic non-epileptic seizures (PNES), functional dystonia and functional movement disorders (FMD); the inauguration of the Functional Neurological Disorder Society in 2017 is testament to recent developments in the field. ${ }^{1}$

Hysteria, conversion, psychosomatism; functional medicine has a history stretching back millennia, and since the days of Janet, Charcot and Freud, psychological trauma has been recognised as key to the aetiology of functional disease. Rigorous new metaanalyses confirm that physical, sexual and emotional abuse, particularly in childhood, predispose to the development of FND with up to $74 \%$ of patients having suffered emotional neglect

Author: ${ }^{A}$ foundation year 1 doctor, NHS Greater Glasgow and Clyde, Glasgow, UK before adulthood. ${ }^{2}$ In his book, The body keeps the score, detailing a life's work treating post-traumatic stress disorder (PTSD), Bessel van der Kolk proclaims that we are on the verge of becoming a trauma-conscious society. ${ }^{3}$ Our newfound openness in confronting this menace is key to why functional medicine has seen such progress in recent times, however, trauma-informed care is still in its infancy and must become more widespread if we are to continue to advance. Gaps in our understanding of how best to treat and diagnose these conditions still exist, leaving much space for budding researchers to make contributions going forward. Even if this isn't your chosen area of study, a generation of aware and understanding doctors would allow these patients to seek the assistance they greatly need.

\section{Aids to diagnosis}

Contemporary theories suggest that among people with FND, high levels of severely stressful life events, especially in the month preceding symptom onset, contribute to abnormalities in the way the brain predicts motor and sensory activity leading to dysfunction. ${ }^{4}$ Previous sensitising factors are thought to determine how a functional disease will manifest; for example, patients with PNES frequently have family histories of epilepsy and may therefore be subconsciously familiar with fitting as a sick role behaviour. ${ }^{5}$ While psychological comorbidity and recent stress are common in FND, these clinical features are also common in organic disease and so caution should be taken when factoring this into a differential. ${ }^{6}$

Once a 'diagnosis of exclusion', recent efforts favour using positive signs to help diagnose and convey a diagnosis of FND. Hoover's sign temporarily elicits extension in a patient's functionally paralysed leg when actively flexing the contralateral hip. Similarly, a tremor that can be 'entrained' to match an instructed rhythm is evidence for it being functional in origin rather than organic. Demonstrating such signs can often encourage patients that improvement of their symptoms is possible and start the path to treatment. "Medically unexplained symptom' profiles which are physiologically impossible (such as sensory loss that cannot be explained anatomically and tubular vision which defies the laws of optics) may also point towards a functional aetiology. ${ }^{7}$

Inconsistency of symptoms can be one of the most useful things to look out for; an abnormal gait present when walking forwards but not backwards, side stepping or running, or alternatively a tremor varying in frequency suggest a functional origin. 6,8 
Incongruity with known neurological diseases can also be assessed but may be best left to specialists.

\section{Under-recognition of FND}

Despite PNES accounting for $20 \%$ of referrals to seizure clinics and functional weakness being at least as common as multiple sclerosis, FND is frequently under-recognised as illustrated by the following case study. ${ }^{7,8}$

\section{Case study}

A 34-year-old woman was brought to the emergency department (ED) by ambulance after she fell to the floor, became unresponsive and began fitting while out with a friend on a busy shopping street. The ambulance crew had already initiated oxygen therapy and given two doses of $4 \mathrm{mg}$ intravenous lorazepam before arriving to the ED. At presentation, vital signs were within normal ranges and a minimal examination revealed tightly shut eyes with apparent lacrimation. There was no obvious tongue biting and limbs continued to jerk asynchronously with arching of the neck and spine. A phenytoin infusion was commenced.

After over an hour of seizure-like activity, the patient became atonic and was able to communicate with doctors. Although drowsy, the patient reported that she had been aware during the seizing. She had experienced similar, shorter episodes of fitting recently and was awaiting an appointment at a 'first-fit' clinic.

This case outlines a common misdiagnosis in the ED; $27 \%$ of patients with PNES have been treated erroneously in intensive care units for mistaken status epilepticus and PNES has seen a consistent average of a 7-year delay to diagnosis. ${ }^{8,9}$ When the correct diagnosis is eventually made, the benefits are obvious, with regular use of emergency medical services dropping from $50 \%$ to $16 \%$ and a significant proportion of patients becoming seizure free following diagnosis alone. ${ }^{9}$

In this case, signs common to PNES and rare in status epilepticus should have been recognised earlier, avoiding the risk of iatrogenic harm from unnecessary antiepileptic medication. PNES may present with intra-ictal resistance to eye opening and crying, reduced or asynchronous limb movements, head shaking or pelvic thrusting. Tongue biting is rare and post-ictal re-orientation is rapid, although in this case, heavy sedation slowed recovery. In a tonic-clonic status epilepticus, the light reflex is lost, movements are rhythmic and stereotyped, respiration is stertorous and recollection of the fit is rare. ${ }^{6-8}$ Prolactin may be raised within 20 minutes of a tonic-clonic seizure with creatinine kinase rising after 2 days. $^{8}$

\section{Learning points}

> Stereotyped tonic phase followed by 'clonic' type jerks are more likely due to epileptic seizures than PNES.

> Resistance to eye opening and intra-ictal crying are specific for PNES compared with epileptic seizures.

> PNES is often followed by a rapid post-ictal recovery.

FMD misdiagnosed as stroke is also common, leading to $9 \%$ of those with functional stroke mimics receiving thrombolysis unnecessarily. ${ }^{10}$ Pronator drift without pronation and Hoover's sign suggest a functional diagnosis, however, these signs are less specific than those for PNES and so imaging available in the acute stroke setting should also be taken advantage of. ${ }^{8}$

\section{What next?}

Knowing where to turn when a functional problem is suspected is the next problem for a junior doctor. In contrast to factitious disease (in which symptoms are voluntarily produced for psychological need) and malingering (a non-disease entity in which symptoms are voluntarily produced for a specified goal), functional magnetic resonance imaging has proven the involuntary nature of functional disorders. ${ }^{11}$ Despite this, these conditions are often confused by medical staff to the detriment of patient care.

Taking a thorough history is useful as patients often experience added disability due to fatigue, pain and problems with concentration on top of their presenting complaint. ${ }^{6}$ Triggers as well as states of mind preceding symptom onset can be explored and generally being a friendly ear can be of use to patients who have often received little sympathy or answers in the past.

A bizarre presentation does not necessarily dictate a diagnosis of FND. A neurologist should be consulted to look for positive signs and to assess the possibility of symptoms being prodromal or existing as a functional overlay to organic disease. Gold-standard methods such as video-electroencephalography can then also be used to diagnose PNES, for example. Misdiagnosis of FND remains extremely rare at $4 \%$, a figure comparable with other neurological and psychiatric conditions. ${ }^{8}$ Specialist services, however, are sparce and provision must improve for earlier recognition of these conditions.

Once a functional diagnosis is made, we as junior doctors will spend the most time with the patient on the ward. If necessary, time can be taken to sensitively educate ward staff and deescalate in times of further psychogenic seizure, for example. The multidisciplinary team can be introduced, which may include physiotherapists, occupational therapists, neuropsychologists and psychiatrists. Importantly, a clear diagnosis must be shared with the patient recognising FND to be real and explaining that it should be treated like any other neurological condition. ${ }^{6}$

A patient's acceptance of a diagnosis is the first step in their treatment and is reported to be prognostic of outcome in FMD by $61 \%$ of professionals. ${ }^{12}$ Pioneered by Prof Jon Stone and Dr Alan Carson in Edinburgh, countless resources exist for both patient and clinician to aid in the sharing of a diagnosis of FND, including comic strips, patient guides and NHS commissioning advice. Neurosymptoms.org may be a good place to start for curious foundation trainees, as dissemination of this information will be key to the advancement of care going forward.

\section{Current state of treatment}

Early and well communicated diagnosis is associated with better outcomes for FND. ${ }^{8}$ However, in order to progress, we must shift from the 'showmanship of a diagnosis' according to Dr Barbara Dworetzky in a recent online Functional Neurological Disorder Society lecture. This sentiment harkens back to Charcot at the Salpêtrière, who would demonstrate his clinical findings to a room of students and peers. Now, Dr Dworetzky argues, treatment must become our focus. Disappointingly, $67 \%$ of patients report symptoms as the same or worse at 1 -year follow-up, suggesting there is much room for improvement in terms of treatment efficacy. ${ }^{8}$

Some headway has already been made into determining the best ways to treat this heterogeneous group of conditions. Specialist physiotherapy has demonstrated improvement in $60 \%-70 \%$ of patients with FMD. ${ }^{13}$ Contrary to typical 
physiotherapy, strength in individual joints are not the focus and whole-body movement is encouraged. Despite all current evidence being favourable, very few NHS trusts provide the specialist physiotherapy services. It is hoped that robust evidence will sway commissioners to recognise the utility of the treatment.

In terms of psychotherapy for FND, cognitive behavioural therapy (CBT) has thus far had the most inquiry. In PNES, the evidence for CBT improving seizure frequency is inconclusive, however, a number of additional benefits have been noted with patients reporting higher overall quality of life, seizures to be less bothersome and lower levels of psychological distress than controls. ${ }^{14}$ Epidemiological factors which could tailor care provision going forward have also been noted in such studies as participants were most often female, unemployed and living in high deprivation areas.

A number of other treatment strategies for FND have seen partial exploration, including hypnosis (which may be more appropriately used as a diagnostic tool) and neuro-feedback (an effective aid in changing illness beliefs). ${ }^{7,13}$ Therapeutic sedation using propofol induction has also shown possible utility as an adjunct to other therapies. ${ }^{8}$

Both thought to be underlain by trauma aetiologies, borrowing from the associated field of PTSD may also prove useful for FND. Possibilities include the use of 3,4-methylenedioxymethamphetamine (MDMA) to assist in psychotherapy and eye movement desensitisation and reprocessing (EMDR) therapy for integrating traumatic memories. $^{3}$

While Bessel van der Kolk argues for a move to see psychiatric diseases as dysfunctions in overlapping brain networks, interrelated and more like symptoms rather than disease entities in and of themselves, Dr Primavera Spagnolo views FND similarly as 'circuitopathies' and is investigating transcranial magnetic stimulation as well as genetic and epigenetic correlates to help target future treatment. ${ }^{3,15}$ It is likely there will be no panacea found for FND. Large studies are necessary to assess the efficacy of all these treatments, requiring interested researchers to carry them out.

\section{Conclusion}

This guide hopefully serves as an introduction to FND for junior doctors and offers tips on recognising and communicating a diagnosis. A generation of doctors acquainted with and able to offer treatments for these trauma-based diseases is needed and findings must be translated across to other specialties. The Functional Neurological Disorder Society aims to start by standardising nomenclature. With over 15 pseudonyms for PNES alone, allowing us to all speak the same language when talking about FND seems like a good next step.

\section{Acknowledgements}

The author would like to make acknowledgement to the Functional Neurological Disorder Society for its excellent series of lectures.

\section{References}

1 American Psychiatric Association. Diagnostic and statistical manual of mental disorders: Fifth edition. Arlington: American Psychiatric Association, 2013.

2 Ludwig L, Pasman JA, Nicholson T et al. Stressful life events and maltreatment in conversion (functional neurological) disorder: systematic review and meta-analysis of case-control studies. Lancet Psychiatry 2018:5:307-20.

3 van der Kolk B. The body keeps the score: Brain, mind, and body in the healing of trauma. Viking, 2014.

4 Keynejad RC, Frodl T, Kanaan R et al. Stress and functional neurological disorders: Mechanistic insights. J Neurol Neurosurg Psychiatry 2019;90:813-21.

5 Vincentiis S, Valente KD, Thomé-Souza S et al. Risk factors for psychogenic nonepileptic seizures in children and adolescents with epilepsy. Epilepsy Behav 2006;8:294-8.

6 Bennett K, Diamond C, Hoeritzauer I et al. A practical review of functional neurological disorder (FND) for the general physician. Clin Med 2021;21:28-36.

7 Espay AJ, Aybek S, Carson A et al. Current concepts in diagnosis and treatment of functional neurological disorders. JAMA Neurol 2018;75:1132-41.

8 Hallett M, Stone J, Carson A (eds). Handbook of clinical neurology: Functional neurological disorders: Vol 139. Elsevier, 2016.

9 McKenzie P, Oto M, Russell A, Pelosi A, Duncan R. Early outcomes and predictors in 260 patients with psychogenic nonepileptic attacks. Neurology 2010;74:64-9.

10 Wilkins SS, Bourke P, Salam A et al. Functional stroke mimics: Incidence and characteristics at a primary stroke center in the Middle East. Psychosom Med 2018;80:416-21.

11 Cojan Y, Waber L, Carruzzo A, Vuilleumier P. Motor inhibition in hysterical conversion paralysis. Neuroimage 2009;47:1026-37.

12 LaFaver $\mathrm{K}$, Lang AE, Stone ] et al. Opinions and clinical practices related to diagnosing and managing functional (psychogenic) movement disorders: changes in the last decade. Eur ] Neurol 2020;27:975-84.

13 Nielsen G, Stone J, Matthews A et al. Physiotherapy for functional motor disorders: a consensus recommendation. J Neurol Neurosurg Psychiatry 2015;86:1113-9.

14 Goldstein LH, Robinson EJ, Mellers JDC et al. Cognitive behavioural therapy for adults with dissociative seizures (CODES): a pragmatic, multicentre, randomised controlled trial. Lancet Psychiatry 2020;7:491-505.

15 Spagnolo PA, Norato G, Maurer CW et al. Effects of TPH2 gene variation and childhood trauma on the clinical and circuit-level phenotype of functional movement disorders. J Neurol Neurosurg Psychiatry 2020;91:814-21.

Address for correspondence: Dr Andrew Whitfield, NHS Greater Glasgow and Clyde, 1055 Great Western Road, Glasgow G12 0XH, UK.

Email: andrew0whitfield@gmail.com

Twitter: @drew_neuro 